\title{
APPLICATION OF CONVOLUTIONAL NEURAL NETWORK IN CLASSIFICATION OF HIGH RESOLUTION AGRICULTURAL REMOTE SENSING IMAGES
}

\author{
Yao Chunjing $^{\text {a }}$, Zhang Yueyao ${ }^{\text {a* }}$, Zhang Yaxuan ${ }^{\text {a }}$, Haibo Liu ${ }^{\mathrm{b}}$ \\ ${ }^{a}$ School of Remote Sensing and Information Engineering, Wuhan University, Luo Yu Road No. 129, Wuchang District, Wuhan \\ City, Hubei Province, P. R. China - zhangyueyao@whu.edu.cn \\ ${ }^{\text {b }}$ State Power Economic Research Institute, Future science and Technology City, Changping District, Beijing City, P. R. China - \\ zhangjiyong@ chinasperi.sgcc.com.cn
}

\section{Commission III, WG III/6}

\begin{abstract}
KEY WORDS: Convolutional Neural Network, Deep Learning, Crop Classification, Agricultural remote sensing, High resolution image
\end{abstract}

\begin{abstract}
:
With the rapid development of Precision Agriculture (PA) promoted by high-resolution remote sensing, it makes significant sense in management and estimation of agriculture through crop classification of high-resolution remote sensing image. Due to the complex and fragmentation of the features and the surroundings in the circumstance of high-resolution, the accuracy of the traditional classification methods has not been able to meet the standard of agricultural problems. In this case, this paper proposed a classification method for high-resolution agricultural remote sensing images based on convolution neural networks(CNN). For training, a large number of training samples were produced by panchromatic images of GF-1 high-resolution satellite of China. In the experiment, through training and testing on the $\mathrm{CNN}$ under the toolbox of deep learning by MATLAB, the crop classification finally got the correct rate of $99.66 \%$ after the gradual optimization of adjusting parameter during training. Through improving the accuracy of image classification and image recognition, the applications of CNN provide a reference value for the field of remote sensing in PA.
\end{abstract}

\section{INTRODUCTION}

\subsection{Background Introduction}

Remote sensing image classification technology is the basic technology of remote sensing scientific, the classification is interpreted by artificial visualization in the beginning. Due to its high labour cost and high uncertainty, computer automatic classification technology has gradually become the mainstream in academic circles. It is divided into supervised classification and unsupervised classification, in order to improve the classification accuracy, scholars put forward the decision tree, fuzzy mathematics, neural network and other new methods in recent years.

With the development of high-resolution remote sensing images, the requirements of classification are getting higher and higher, the classification of complex scenes and the requirements of rotation translation invariance give birth to a series of studies on the deep learning in the field of remote sensing. Among them, convolution neural network $(\mathrm{CNN})$ is particularly significant.

At the same time, with the rapid development of Precision Agriculture (PA) promoted by high-resolution remote sensing, it makes significant sense in management and estimation of agriculture through identification of high resolution remote sensing image of crop.

In this paper, the convolution neural network $(\mathrm{CNN})$ is adapted to classification and extraction of crops in the environment of high-resolution agricultural remote sensing images.

\subsection{Previous Work}

For the classification of high-resolution images, many scholars have made research: Jie Chen used object-oriented classification method to study high-resolution remote sensing images. In addition, there are improved support vector machines, semantic algorithms and other new methods.

Now, more and more people began to study the application of machine learning in various fields. In the field of image processing, neural networks are widely used, especially convolution neural network. Xinchang Gao, Du Jing, Dawei Liu and others use deep learning to classify remote sensing images. Castelluccio used the convolution neural network to classify land use. Linlin Cao used convolution neural network model for image classification.

\section{CONVOLUTIONAL NEURAL NETWORK}

\subsection{General Instructions}

Over the past few years, deep learning has performed well in solving many problems, such as visual recognition, speech recognition and natural language processing. Among the different types of neural networks, the convolution neural network is the most deeply studied.

In the 1960s, Hubel and Wiesel found that cat's unique network structure can effectively reduce the complexity of the feedback neural network when studying the neurons in the local sensitive and directional selection of the cortical cortex. Then, the convolution neural network $(\mathrm{CNN})$ was put forward. $\mathrm{CNN}$ has become one of the hotspots in many fields of science recently, especially in the field of pattern classification. Since 
the network avoids the complicated pre-processing of images, it can be input the original image directly.

The basic structure of CNN consists of two layers, one for the feature extraction layer, and each neuron is connected to the local window of the previous layer. The second is the feature mapping layer. Each calculation layer of the network is composed of multiple feature maps. Each feature map is a plane, and the weights of all the neurons on the plane are equal.

CNN has a unique superiority in speech recognition and image processing with its special structure by shared local weights, its layout is closer to the actual biological neural network.

\subsection{Local Perception}

Each neuron senses a local image, and then integrates the local information at a higher level to obtain global information.

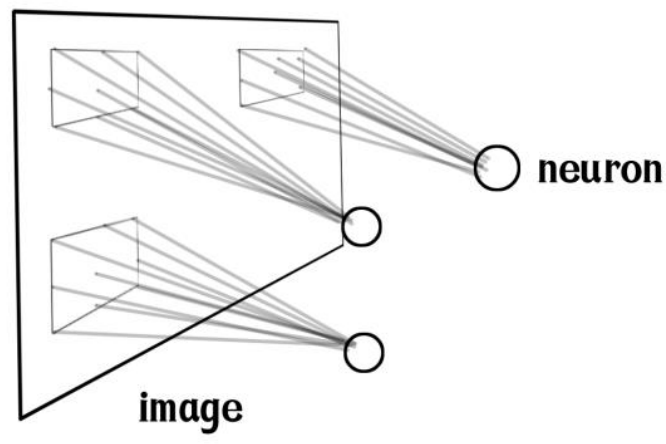

Figure 1. Local Perception of CNN

\subsection{Shared Weight}

When convolution is performed on an image, it is not necessary to create a new parameter for each convolutional kernel, and the convolution kernel parameters in the sliding process are shared. The weight of this process is called shared weight, the bias of this process is a shared bias. Shared weight with shared bias is a convolution kernel or filter.

\subsection{Pooling}

Pooling is an operation to find out whether there is a feature location in the image area. After the image feature position is found, the position information of the feature can be discarded. The pooling layer can reduce the number of parameters during the next operation.

\section{Convolved Feature}

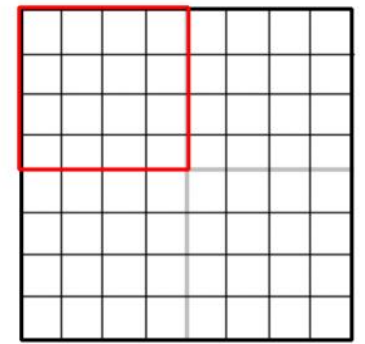

\section{Pooled Feature}

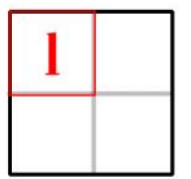

The above are the basic network layers, in the training process we need to gradually adjust the parameters to adapt to the data set.

\section{EXPERIMENT AND ANALYSIS}

In order to verify the reliability of crop classification using convolution neural networks, a 11-layer convolution neural network is constructed, including the input layer, three convolution layers, two pooling layers, two local contrast adjustment layers, two full connection layers and one output layer. The structure of the convolution neural network is as follows:

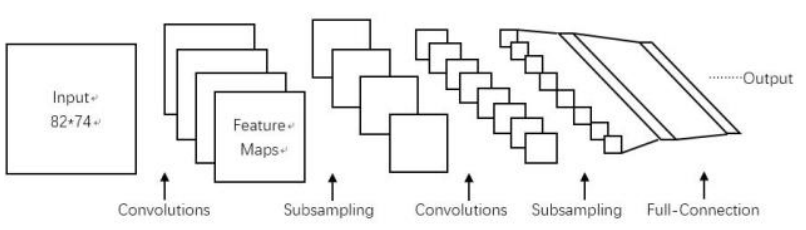

Figure 3. Structure of CNN

\subsection{Test Data Set}

In this paper, we use a large number of training samples produced by panchromatic images of GF-1 high resolution satellite of China with $2 \mathrm{~m}$ resolution, to visually classify the crop images of, Ezhou, Hubei. Image acquisition time is May 12, 2016.

Taking the strong temporal characteristics and regional characteristics of crop into account, this article makes a basic understanding of the crop cultivation in Ezhou: Ezhou's grain crops are mainly rice, cash crops mainly rape, also planting cotton, lotus root and little other crops. In mid-May, Ezhou rice get into the heading and stooling stage, growing well, the rape has been the end of a comprehensive harvest, cotton is in the seedling period between the flowering period, and lotus root is in the period of pumping leaves.

Combined with the image feature of various types, the category -label-image correspondence table is as follows:

\begin{tabular}{|l|l|l|l|l|l|l|l|l|}
\hline Pond & Rice & Algae & $\begin{array}{l}\text { Waste- } \\
\text { land }\end{array}$ & River & $\begin{array}{l}\text { Build- } \\
\text { ing }\end{array}$ & Wood & Road & $\begin{array}{l}\text { Plant } \\
\text {-ing }\end{array}$ \\
\hline 0 & 1 & 2 & 3 & 4 & 5 & 6 & 7 & 8 \\
\hline 7 & & & & & & & & \\
\hline & & & & & & & & \\
\hline
\end{tabular}

Table 1. Category-Label-Image Table

According to the correspondence table, through repeated manual identification, 1,500 basic data sets were created, then a total of 6,000 data sets were obtained after the rotation operation, $80 \%$ of which was used as training CNN, $20 \%$ was used for verification. In order to distinguish feature images, the pixel size of each image is $82 * 74$.

Figure 2. Pooling Layer of CNN 


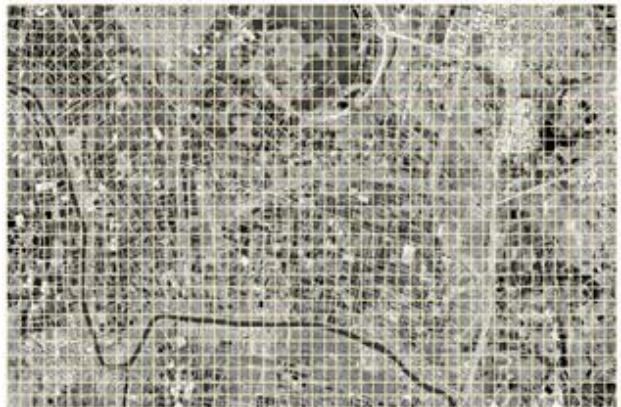

Figure 4. Training Data

\subsection{Analysis of Test Results}

After the gradual optimization of adjusting parameter during training, the final parameters are as follows:

\begin{tabular}{ll}
\hline Convolution core & $\mathbf{7} * \mathbf{7}$ \\
\hline Learning Rate & 1 \\
\hline Batchsize & 50 \\
\hline Training Times & 1 \\
\hline Activation Function & sigmoid \\
\hline
\end{tabular}

Table 2. Parameters of CNN

The crop classification finally got the correct rate of $\mathbf{9 9 . 6 6} \%$. In addition to judge the classification accuracy, this paper also draws a loss function to describe the error between the estimated value and the true value projected to a certain feature space.

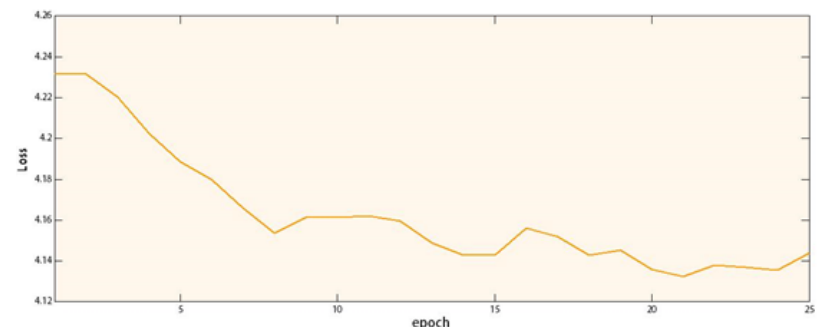

Figure 5. Loss Function

In order to avoid the phenomenon of supersaturation, this paper uses the ReLu function to compare the results. ReLu makes the output of some neurons 0 , resulting in the sparseness of the network, and reducing the interdependence of parameters, finally alleviating the occurrence of supersaturation problems. The following table lists the effect of the two activation functions.

\begin{tabular}{|c|c|c|c|}
\hline Function & Formula & Curve & $\begin{array}{c}\text { Correct } \\
\text { rate }\end{array}$ \\
\hline sigmoid & $\Theta(x)=\frac{1}{1+e^{-x}}$ & & $99.66 \%$ \\
\hline ReLu & $\mathrm{F}(\mathrm{x})=\max (0, \mathrm{x})$ & & $88.87 \%$ \\
\hline
\end{tabular}

Table 3. Comparison of Two Activation Functions

Through the comparison of supervision classification and unsupervised classification by remote sensing software, it was found that the convolution neural network method has incomparable high precision. At the same time, the convolution neural network model has a great effect in reducing the errors caused by image translation, zoom, tilting, or other forms of deformation.

\subsection{Comparison of Experimental Results}

In order to verify the effectiveness of $\mathrm{CNN}$, this article uses other classification methods for comparative experiments. The experimental data were classified based on support vector machine (SVM) and supervised classification based on parallelepiped, unsupervised classification. In addition, multiband images were classified by supervised classification based on maximum likelihood.

It can be seen that $\mathrm{CNN}$ results are better than existing methods. Among these classification methods, the supervised classification and unsupervised classification based on panchromatic images have obtained poor results, on the other hand, as the same classification parameters, the supervised classification based on multi-band images results are better, we can see that the requirements of multi-spectral data for the supervised classification.

\begin{tabular}{|l|c|c|}
\hline \multicolumn{1}{|c|}{ Methods } & $\begin{array}{l}\text { Overall } \\
\text { Accuracy }\end{array}$ & $\begin{array}{c}\text { Kappa } \\
\text { Coefficien } \\
\mathrm{t}\end{array}$ \\
\hline $\begin{array}{l}\text { Supervised } \\
\text { (panchromatic } \\
\text { images) }\end{array}$ & $14.7 \%$ & 0.06 \\
\hline $\begin{array}{l}\text { Supervised } \\
\text { (multi-band } \\
\text { images) }\end{array}$ & $87.66 \%$ & 0.85 \\
\hline unsupervised & $44.6 \%$ & 0.32 \\
\hline \multicolumn{1}{|c|}{ SVM } & $80.7 \%$ & \\
\hline CNN & $88.87 \%$ & \\
\hline
\end{tabular}

Table 4. Different Classification Results

\section{CONCLUSION}

Through improving the accuracy of image classification and image recognition, the applications of convolution neural network provide a reference value for the field of remote sensing in precision agriculture(PA).

For CNN, a large number of training data sets are the basis for accurate, so expanding the data set is a direction of improving this article furtherly. In the future study, if the multi-temporal characteristics of crop remote sensing images can be added to the convolution neural network training, the prediction may get good results, and this also put forward a new direction for multi-temporal image extraction.

\section{REFERENCES}

Castelluccio M., Poggi G., Sansone C., 2015a. Land ues classification in remote sensing images by convolutional neural networks. Journal of Molecular Structure Theochem, 537(1), pp. 163-172.

Linlin Cao, 2016a. Application of convolutional neural networks in classification of high resolution remote sensing imagery. Science of Surveying and Mapping, 2016(9), pp. 170175 
Changxin Gao, Nong Sang, 2014a. Deepleaarning for Object Detection in Remote Sensing Image. Bulletin of Surveying and Mapping, 2014(S1), pp .108-111.

Guanyu Chen, Kai An, Xiang Li, 2016a. Identification and Classification of Bad Geological Body Based on Convolution Neural Network. Geological Science and Technology Information,35(1), pp.205-211.

Xiaofei He, Zhengrong Zhou, Chao Tao, 2016a. Jiaxing Zhang. Combined Saliency with Multi-Convolutional Neural Network for High Resolution Remote Sensing Scene Classification. Acta Geodaetica et Cartographica Sinica, 45(9), pp.1073-1080.

Dawei Liu, Ling Han, 2016a. High Spatial Resolution Remote Sensing Image Classification Based on Deep Learning. ACTA OPTICA SINICA, 36(4), pp.306-314.

Jing Du, 2017a. Deep Learning Based UAV Remote Sensing Image Water Body Identification. JIANGXI SCIENCE, 35(1), pp.158-161+170.

Shuang Zhao, 2015. Remote Sensing Image Classification Method Based on Convolutional Neural Networks. China University of Geosciences.

Baojian Xie, 2015. The Research of Image Classification Methods Based on Convolutional Neural Network. Hefei University of Technology.

Minnan Chu, 2015. Research of Image Classification Technology Based on Convolutional Neural Network. Xiangtan University.

Jie Chen, 2010. Study on Object-based Classification of HighResolution Remote Sensing Imagery. Zhongnan University. 